\title{
Analysis of urban spatial cognition in Luzhou city based on city image theory
}

\author{
Lin Lu, Peijia Jiang, Xu Zhang, Yu Zhao \\ College of Landscape Architecture, Sichuan Agricultural University, No.211, Huimin Road, Wenjiang District, Chengdu, China
}

\begin{abstract}
This paper uses the methods of picture recognition, sketch map and questionnaire to understand the citizens' evaluation of Luzhou's urban environment, obtain the overall city image and influencing factors to explore the spatial composition and city characteristics of Luzhou.Based on the analysis of the deficiencies in the process of urban development, this paper puts forward some appropriate suggestions to construct a good city image of luzhou city space.
\end{abstract}

\section{Introduction}

City imagery ${ }^{[1]}$ is a thought of urban planning and design proposed by Kevin Lynch after thorough research and analysis of cities such as Boston, Jersey City and Los Angeles.The "city image"is a pioneer in modern city space research by introducing the psychological factors of citizens instead of the previous seemingly objective academic composition rules.

While luzhou is located in the south of sichuan province, is the "Chinese famous historical and cultural city", "National Double Support Model City", "National Health City", "Sichuan Province Social Security Comprehensive Management Model Area", with outstanding location advantages and geographical conditions, is the commercial center and important material distribution center in the junction of Sichuan, Yunnan, Guizhou and Chongqing. This paper investigates the image of Luzhou,aiming to provide the basis for urban design, establish a good city image space to bring enough attraction for citizens and create a "safe, convenient, comfortable and distinctive" city pattern.

\section{The research methods}

This study uses picture recognition,sketch $\operatorname{map}^{[2]}$ and questionnaire $^{[3]}$ to analyze the city image of Luzhou. The picture recognition is the first step of the survey.We selected 30 points from the map of Luzhou and took 80 photos of landscapes and buildings to these points through field investigation. Select 40 of them and conduct a small sample survey. The small sample was 10 people, all luzhou residents who had lived in Luzhou for more than 10 years. Then selected 26 photos that can be recognized by more than 10 people from 40 photos and numbered as the photos used in the survey.Then the investigators presented the photos to the respondents and asked them to say the name or the general location of the scene in Luzhou. One of the two answers was correct, and put the picture aside. Finally, numbered the photos recognized by the respondents and mark them on the questionnaire (Figures 1).The second method is the sketch map. Respondents need to outline a simple map of their city(Figure 2). Such a simple map of the cognitive city is called the "Sketch Map", which used to obtain data on how people perceive the environment.For the convenience of research, this paper uses the term cognitive map and limits the concept of cognitive map to "People's perception of imagery".
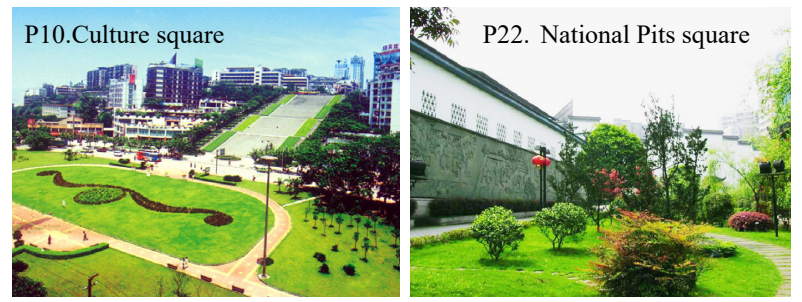

Fig. 1. Picture recognition

The basic data of the survey consists of photo identification data, cognitive map data and questionnaire data.A total of 122 questionnaires were issued in the survey that obtained photo data, and the effective rate of the questionnaires was $82 \%$.Cognitive map data was obtained by asking respondents to draw a map of Luzhou. Questionnaire interview data were obtained by filling in a questionnaire(survey form).
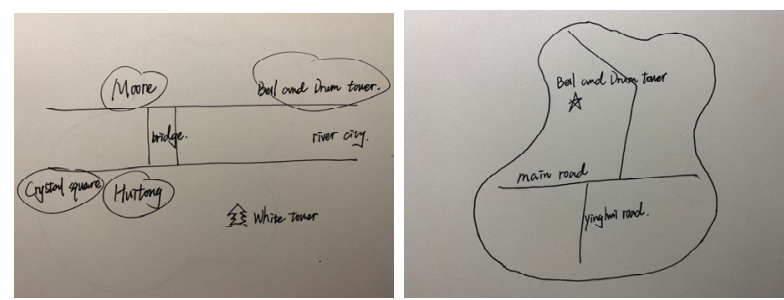

Fig. 2. Sketch map 


\section{The results of survey}

\subsection{The results of picture re-read}

The 26 pictures were divided into 7 categories according to their functions,namely landmark buildings, leisure and entertainment places,commercial places,polpulation center,cultural and sports places,scenic spots and political landmarks. The overall identification of photos by the social group is shown in Table 1 .

\subsection{Sketch map}

We received 33 sketch map.According to the scope of the cognitive map drawn by the respondents, it can be divided into four categories(Figure 3).According to the analysis of the sketch map,the city image is based on the pattern of common interaction between city and people. Because people have the closest interaction with the region where they live and work,the sketch map is mostly based on the starting point or scope of residence and work place,and is more detailed than other
regions.Commercial centers are closely related to the daily life of citizens that have more opportunities to contact with them,so their intentionality is also very high.

The most common element in the sketch map is the road,which shows that the road is the main frame of Luzhou's spatial image. It plays a role in connecting other elements and helping people to conduct spatial location.Commercial buildings, such as Huitong - Moore and other large shopping malls, appear more frequently in the logo elements, followed by buildings with certain external features (such as bell and drum towers). The boundaries of Luzhou Administrative district are very obvious on the map, but administrative districts appear very rarely in sketch map, which are significantly lower than commercial districts and residential districts.Due to the special geographical location of Luzhou, the appearance of boundaries is mostly combined with rivers and paths.Thus,it can be seen that the macro spatial structure image of luzhou is supported by the main roads, with the commercial district and residential area as the background,and the three-dimensional frame structure as the characteristic bulidings.

Table.1.Photo identification number statistics

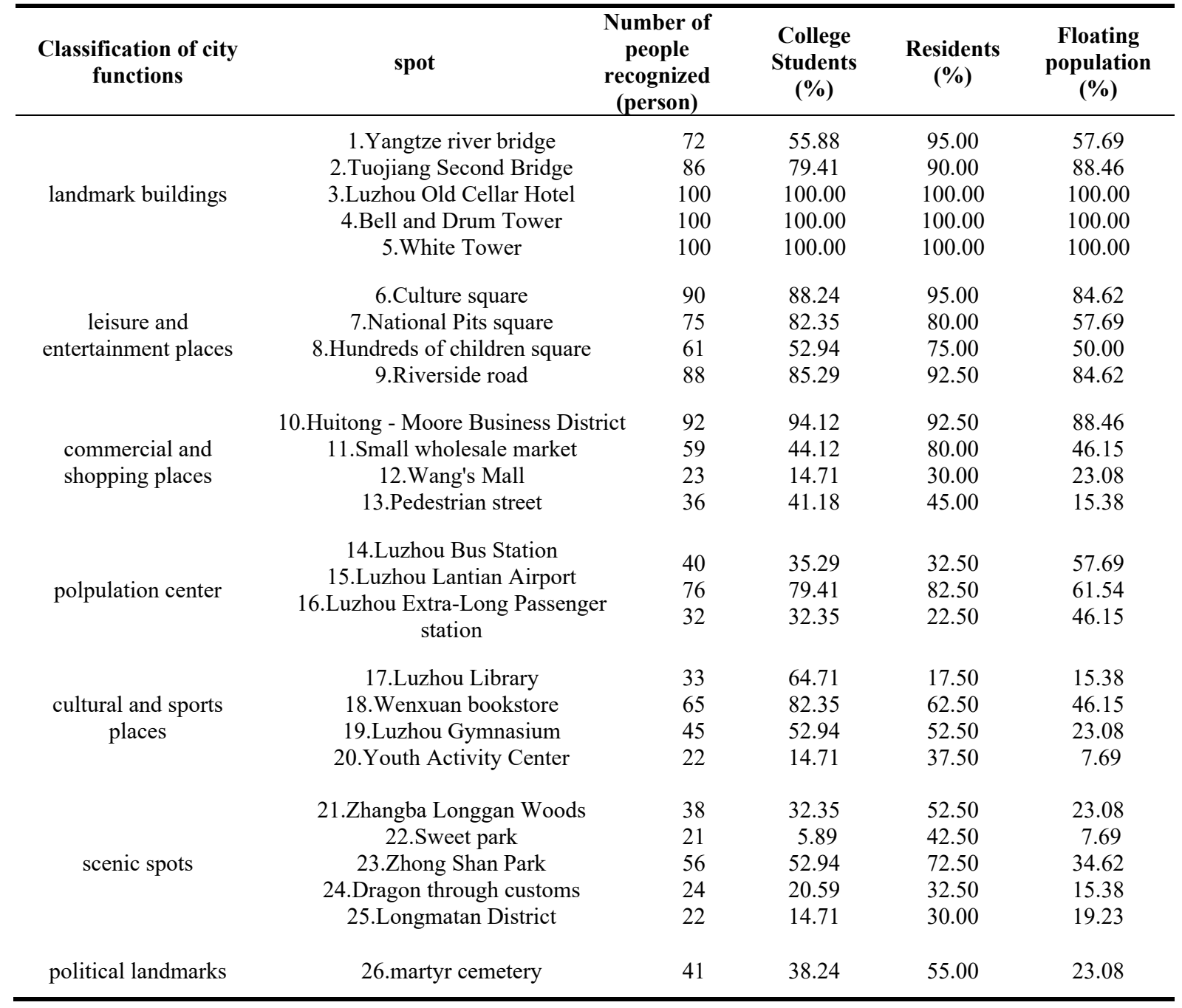




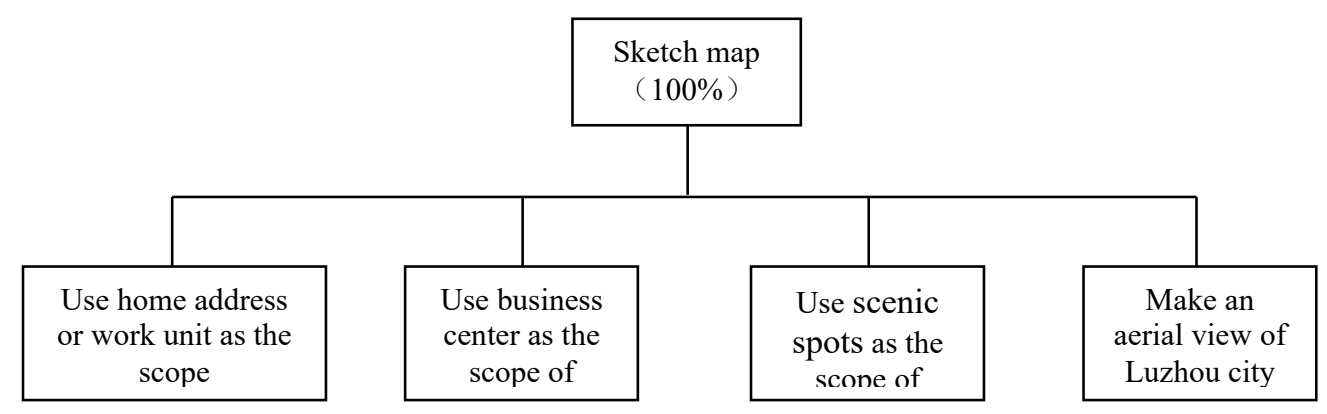

\section{Image analysis of Luzhou}

Fig.3.Sketch map range classification map most representative ones are the National Pits square,Riverside road and the Bell and drum Tower.

\subsection{Analysis of Image elements}

(1).Path and edge.The Yangtze River and Tuojiang River are not only the waterway, but also the natural boundary of the town, which has become the basic reference system for residents to remember the urban environment.All main roads are parallel or perpendicular to the Yangtze River or tuojiang River, which is conducive to the judge the general direction. At the same time, they also play the role of traffic passage and boundary,together with the Yangtze River and Tuojiang River, constitute the urban cognitive structure of the whole Luzhou City.

(2).District.Luzhou is located at the confluence of the Yangtze River and the Tuojiang River. The two rivers clearly divide the city into three regions -Shizhong District (part of Jiangyang District), Jiangyang District and Longmatan District.Therefore, respondents are familiar with the environment of their own region, while the other two regions are relatively vague. In the public image of the whole city, the occurrence rate of all city elements is not very high.

(3).Node and landmark.As the direction of the Yangtze River and The Tuojiang River determines the direction of city roads, the respondent does not have a clear sense of direction like northerners,so the nodes and markers play a more prominent role in the identification of the environment.

Riverside road constitutes the largest node in Shizhong District.It is built along the river, where the Tuojiang River merges into the Yangtze River,the scene is spectacular.Although the respondents' memory of the city were more focused on the area where they were located, their memory of the environment in this area was particularly clear.Almost all of the environmental elements that appear most frequently in public imagery are in this area.Among them,Riverside road is $88 \%$, Luzhou Old Cellar Hotel is $100 \%$, Yangtze River Bridge is $72 \%$, Tuojiang Bridge is $86 \%$, culture Square is $90 \%$.

In addition,the city's landmarks include the White Tower,the Bell and Drum Tower, the National Pits square, and the Huitong-Moore Business Center.Although Luzhou is a medium-sized city, the number of nodes is relatively small,but from the image statistics, most of the nodes have clear meaning. The

\subsection{Analysis of evaluative image of Luzhou}

The first question of the interview is to obtain people's overall evaluation of Luzhou(Note: The first question is "When you see the word'Luzhou', what do you think of first?")and the main factors influencing people's evaluation image of Luzhou.The answer to this question can be divided into three types:(1)"Wine city", 56 people answered; (2)one or several symbolic places (buildings) in Luzhou,among which Riverside road is the most, 22 people answered, others are bell and drum tower,Luzhou Old Cellar Hotel; (3) food categories,such as "longan", "tofu fish","The piglets dumplings"and so on, are relatively few (1-3 of each kind) and are not representative. Some people also answered both the first and second types of answers.

The second, third, fourth, and fifth questions in the interview include which places in Luzhou are most liked and dissatisfied with. The investigation shows that the factors affecting the overall evaluation image of Luzhou are mainly economic, cultural, traffic and environmental,and also influenced by security and belonging.

\subsection{Investigation on city characteristics of Luzhou}

City characteristics is an image that people acquire through the experience and perception of space and social activities.

In order to obtain information about Luzhou's characteristics from the public,we have designed two questions: "What would you introduce to someone who has never been to Luzhou"and "Which location do you like most in Luzhou". These two questions are significantly different (the coincidence rate is about $25 \%$ ). The reason is that residents are more inclined to answer "The symbol of Luzhou" for the former while "personal preference" for the latter.On the whole,the former more reflects the overall image, while the latter more reflects the diversity of images, that is, individual's different feelings towards the city.Combining two questions, the answers can be divided into three categories: (1) It clearly points out some places and buildings, such as Riverside road, the National Pits 
square, Bell and Drum Tower, white Tower, etc.It is worth pointing out that most of the locations, places, and buildings mentioned by the respondents are landmarks, nodes or areas that have an impact on the structural images, and new commercial districts are the main ones(Table 2).(2)General term for a type of place,such as: historical sites, scenic spots, etc. (3) Non-spatial characteristics,such as economic development, local customs,cultural atmosphere, etc.Among the non-spatial characteristics,"wine culture" was mentioned most,while some of them also mentioned history and food culture.

Table.2. Luzhou influences the place and architecture of critical images

\begin{tabular}{cc}
\hline Place name & number \\
\hline Riverside road & 35 \\
Zhangba & 28 \\
National Pits square & 22 \\
White Tower & 15 \\
Fang mountain & 10 \\
Bell and Drum Tower & 8 \\
Huitong - Moore & 8 \\
Yang Qiao reservoir & 5 \\
\hline
\end{tabular}

\section{Analysis of formative factors affecting urban intention evaluation of Luzhou}

Through this investigation and analysis of Luzhou image space, we can get the conclusions:Economy, history, cultural atmosphere, transportation and environment are important factors that affect the residents' judgmental images $^{[4]}$. There are great differences in the image space of different social groups.

In general,the public in Luzhou has similar structural image of the city, that is, there is a "public image".The main structure of "public image" in Luzhou is clear,and the main factors are roads, landmarks, nodes and cultural atmosphere.The Yangtze River and Tuojiang River are not only the waterway, but also the natural boundary of the town, which has become the basic reference system for residents to remember the city environment.All the main roads are parallel or perpendicular to the Yangtze River or The Tuojiang River, and they also play the role of traffic passage and boundary, which together with the Yangtze River and the Tuojiang River constitute the city cognitive structure of Luzhou.In the immaterial image, the century-old wine culture supports the whole charm of this city. In this way, both inside and outside image have created a delicate, characteristic riverside wine city.

\section{Suggestions on constructing good city image}

As a considerable carrier of a city,the author believes that there are still some deficiencies in the city image of Luzhou.

First of all, the roads in Luzhou are very efficient,but lack individuality. The importance of the road is not only to provide the main visual observation point, but also become the direct perception of city image. The overall perception of roads in Luzhou has a strong sense of direction, but there are still many areas to be improved. For example, there is almost no street furniture on both sides of the main road, no small landscape belt and space for pedestrians to rest; no cultural sculptures and cultural relics, also no road landscape for appreciation. The single function and low recognizability lead to street lacks the cultural perception of city life, which turn into purely vehicular traffic roads.

Second, the city nodes lack the overall appearance.Node is the spatial focus of the city with the meaning of connection and concentration. A node can be the core of area, a central square, a central garden, or a building in a central location.In Luzhou,such as Hundreds of children square, Bell and Drum Tower, White Tower, Luzhou Old Cellar Hotel are the most image nodes, however,due to their single architectural form and function,they can't be the center of city image ,nor can they be composed together,so the city lacks an overall style that can be imaged by people.Therefore, from the perspective of nodes,Luzhou is a continuous city complex that is difficult to remember.

Based on the above analysis, the author puts forward the following Suggestions for planning,and city management of Luzhou:

First, the road pattern of Luzhou not only inherits the traditional road structure, but also adapts to the characteristics of modern city, playing a decisive role in the formation of city image. Therefore, no matter whether it is expansion or redevelopment of the city, this pattern should not be broken.Instead, focus on the cultural construction of the road itself.

Second, according to the obvious characteristics of marginal elements in Luzhou image,the marginal elements should be more prominent in areas with favorable natural conditions. As for the "inner" and "outer" city pattern divided by river, the difference is conducive to the formation of the "outer" city image and the shaping of the "inner" city image, the author suggests to preserve rather than bridge this boundary.

Third, combine nodes and highlight the characteristics of the city.In the process of characteristic investigation, the image that wine culture as a symbol of Luzhou has been rooted in the hearts of Luzhou citizens.However,high imagery and integrity of nodes become the biggest contradiction in city construction,so how to reflect the characteristics and continuously increase the image elements in the nodes has become the focus of node planning.

\section{References}

1. K.Lynch.The image of the city[M].Beijing: Huaxia Press,(2001).

2. X.M.Li,J.H.Li.Analysis of Urban Space in Dalian[J].Acta Geographica Sinica.61,8(2006)

3. J.Feng.Spatial Cognition and the Image Space of Beijing's Residents[J].Scientia Geographica Sinica.25,12(2005)

4. Y.R.Shen.City characteristics and city image[J].Urban Problems,03(2004) 